\title{
Gibt es spezifisch lyrische Äußerungsstrukturen?
}

\section{Anmerkungen zum Verhältnis von Redekriterium und Lyrikbegriff in der jüngeren Lyrikologie}

Definitionen der Lyrik sind häufig (nicht immer) eng verknüpft mit der Frage, was oder wer die lyrische Rede konstituiert. Heinz Schlaffer zum Beispiel legt nahe, die Lyrik als ursprüngliche priesterliche Anrufung höherer Mächte zu betrachten. Eine Anrufung, die in der Moderne zu einem »Selbstgespräch « geworden sei, »dem das ausgeschlossene Publikum verehrungsvoll« lausche (Schlaffer 2012, 198), wenn auch häufig verständnislos. Das ist eine ziemlich konkrete Vorstellung davon, wie ein lyrisches Gedicht nicht nur durch eine Äußerungsstruktur, sondern gar durch eine spezifische Haltung des `Sprechers « bzw. des Adressanten konstituiert wird. ${ }^{1}$ Eine spezifische Form des lyrischen Adressanten postulieren auch Jackson und Prins im Lyric Theory Reader, einem wichtigen US-amerikanischen Sammelband zur Lyriktheorie: Mit lyrischem Gedicht werde seit dem zwanzigsten Jahrhundert eine literarische Produktion bezeichnet, die gelesen und nicht gesungen werde und hauptsächlich eine Aussage über persönliche Gefühle in der ersten Person Singular repräsentiere:

\begin{abstract}
As a term derived from ancient Greek to designate a song accompanied by the lyre, its association with musical performance persists today in popular `song lyrics` with instrumental accompaniment, but >lyric poetry is also invoked more abstractly as a literary production that is read not sung. Often a poem is considered lyric when it presents an utterance in the first person, an expression of personal feeling, according to a model of modern lyric reading that diverges from the way poems were performed (and read) in antiquity. (Jackson und Prins 2014, 1)
\end{abstract}

Es gäbe viel an dieser pauschalen und wenig differenzierten Beschreibung von slyric zu kritisieren, aber das würde an der Zielsetzung der beiden Autorinnen vorbeiführen. Sie erheben gar nicht den Anspruch, eine dem Gegenstand angemessen Definition zu liefern. Vielmehr möchten Jackson und Prins eine zunehmende Universalisierung einer solchen Vorstellung von Lyrik auf eine Vielfalt von Gedichtformen wie Hymne, Ode oder Sonett angreifen. Ziel ihrer Ausführungen

1 Zur Begrifflichkeit sei auf das Glossar (S. 25-50) verwiesen.

Ә Open Access. (C) 2019 Ralph Müller, publiziert von De Gruyter. (c) BY-NC-ND Dieses Werk ist lizenziert unter der Creative Commons Attribution-NonCommercial-NoDerivatives 3.0 Lizenz. https://doi.org/10.1515/9783110520521-005 
ist also eine Kritik des Prozesses der »lyricization of poetry« (Jackson und Prins 2014, 7; Jackson 2005, 6). Dieser Prozess - ich übersetze ihn im Folgenden als >Lyrifizierung` - bezeichnet weniger eine Gattung als eine Auffassung, die unterschiedlichen Gedichtformen eine bestimmte Form von 'Sprecher unterstelle, der über eigene Gefühle aus einer Ich-Perspektive sspricht` (und keinesfalls >singt<).

Auf den ersten Blick hat man es hier mit einer Neuauflage einer Position zu tun, die zum Beispiel auch schon von René Wellek in den 1960er Jahren vorgebracht worden war (vgl. Wellek 2014 [1967], 51). Wellek hatte empfohlen, vom Studium der notorisch schwer zu definierenden Lyrik-Gattung künftig abzusehen und nur noch Gedichtgenres in der Breite ihrer Formen zu betrachten. In dieser Hinsicht bezeichnet `Lyrifizierung` bei Jackson und Prins die Ausweitung des Begriffs >Lyrikı. Vergleichbare Entwicklungen finden sich bekanntlich in der deutschen Gattungspoetik. So war »Lyrica « bei Opitz lediglich eine Gattung neben anderen wie Sylven oder Hymnus (Opitz 1977, [36]). Die allgemeine Aufwertung zu einer Großgattung neben Drama und Epik geschah erst in den letzten zweihundert Jahren - mit allen Definitionsschwierigkeiten, die aus diesem Prozess erfolgen. ${ }^{2}$ Jedoch zielt die Stellungnahme von Jackson und Prins insbesondere auf eine Frage der Lyrik-Interpretation ab. So hat Jackson ihre Argumentation erstmals im Zusammenhang der angemessenen Kontextualisierung von Emily Dickinsons Gedichten vorgebracht. Dickinsons Gedichte sind hauptsächlich posthum publiziert worden und wurden in der daran anschließenden Rezeption vielfach von ihren zum Teil ungewöhnlichen Entstehungs- und Überlieferungszusammenhängen abstrahiert (vgl. Jackson 2005). Und so ist der Hauptvorwurf von Jackson, dass in der Nachfolge des New Criticism und des Poststrukturalismus unterschiedliche Texte als `Lyrikı unabhängig von ihren ursprünglichen Entstehungs- und Gebrauchskontexten gelesen werden (vgl. Jackson 2005, 39).

Bei aller Unzulänglichkeit von Jacksons Begriffsbestimmung der Lyrik scheint gerade dieser Kritikpunkt interessant zu sein für die Lyrikologie, nämlich der Vorwurf, dass ein landläufiges Verständnis von Lyrik eine `Selbstaussprache` eines von allen Kontexten entledigten Adressanten impliziert. Dabei muss man

2 Da sich die Gattungstrias von Epik, Lyrik und Dramatik erst im neunzehnten Jahrhundert als systematische Einteilung der Literatur durchgesetzt hat, bleiben Rückbezüge auf frühere Ausformulierungen etwa in der italienischen Renaissance (vgl. aber Huss et al. 2012) oder gar in Platons Politeia umstritten (vgl. Primavesi 2008, 22-23). Wo auch immer sich ähnliche Konzepte oder Einteilungen andeuten, stehen sie jeweils in einem anderen Gattungssystem und stellt sich jeweils grundsätzlich die Frage, inwiefern diese Aussagen für eine transhistorische Argumentation genutzt werden können. 
die Perhorreszierung des Lyrikbegriffs durch Jackson und Prins nicht unbedingt übernehmen, man kann ihre Stellungnahme auch als Herausforderung für die Lyriktheorie verstehen. Hier setzt auch das Interesse dieses Beitrags an: Wie die nachfolgende Diskussion ausgewählter theoretischer Arbeiten zeigen soll, orientieren sich tatsächlich viele gegenwärtige Definitionen von Lyrik mehr oder weniger offen an einem Adressantenmodell, indem sie zum Beispiel eine notwendige Fiktionalisierung der Gattung aufgrund einer Vermittlungsstruktur setzen oder die Lyrik aufgrund einer besonderen Redehaltung des Dichters bestimmen. Insofern findet sich dabei auch häufig die Auffassung, dass ein lyrisches Ich, der Sprecher (oder welche Instanz auch immer in der Position des lyrischen Adressanten postuliert wird) notwendigerweise vom Autor unterschieden werden muss. Diese Annahme wird im Folgenden als >dekontextualisierter Adressant betrachtet. Die Annahme eines dekontextualisierten Adressanten kann, wie gezeigt werden soll, zwei Konsequenzen haben: Sie kann sich zum einen auf die Definition von >Lyrikı auswirken. Zum anderen kann sie als Interpretationsregel mehr oder weniger differenziert auf eine Vielzahl von unterschiedlichen Texten angewandt werden. Die letzte Option scheint mir dabei, mit Verlaub, die schlechteste zu sein. Während man durchaus Gründe zum Beispiel für eine Differenzierung von `Gedicht ` und `Lyrik ` vorbringen könnte und diese Differenzierung auf der Grundlage eines spezifischen Adressanten vornehmen könnte (auch wenn dies meines Erachtens gegen den im deutschen Sprachraum aufgrund der Gattungstrias vorherrschenden breiten Lyrikbegriff geht), wäre eine grundsätzliche Unterstellung eines dekontextualisierten Adressanten in vielfältigen Gedichtformen ein Verlust an theoretischer und interpretatorischer Differenzierung.

Die in diesem Band vorgeschlagene Begrifflichkeit eines Adressanten reiht sich ebenfalls in diese Diskussion ein. Sie wird in diesem Beitrag aufgegriffen, um unterschiedliche neuere theoretische Beiträge zu lyrischen Äußerungsstrukturen, insbesondere von Petzold (2012), Hempfer (2014) und Culler (2015), zu rekonstruieren. >Adressant eignet sich gerade deswegen für eine solche Rekonstruktion, weil gerade dieser Begriff keine Festlegung auf spezifische lyrische Äußerungsstrukturen impliziert, da lyrische Gebilde prinzipiell auch adressantenfrei sein können.

\section{Lyrismus und Lyrik in der Gattungstrias}

Der enge Lyrikbegriff von Jackson und Prins (2014) scheint in Teilen dem zu entsprechen, was in der französischsprachigen Theorie bisweilen despektierlich als >Lyrismus bezeichnet wird und wesentlich durch die Vorstellung einer gefühlvol- 
len, fast exaltierten Selbstaussprache in Versen geprägt ist. ${ }^{3}$ Das Lyrismus-Konzept lehnt sich an Vorstellungen des neunzehnten Jahrhunderts an. ${ }^{4}$ Beispielhaft kann für diese Konzeption ebenso die in der anglo-amerikanischen Forschung nachhaltig einflussreiche Bestimmung der Lyrik als sabgelauschtes Selbstgespräch` (`soliloquy overheard`) durch den Philosophen John Stuart Mill genannt werden:

Wenn man aber eine solche Antithese gestattet, so möchten wir sagen, daß Beredtsamkeit gehört, Poesie belauscht wird. Beredtsamkeit setzt eine Zuhörerschaft voraus; die Eigenthümlichkeit der Poesie scheint uns dagegen darin zu liegen, daß der Dichter gar keine Ahnung von der Anwesenheit eines Zuhörers hat. Poesie ist Gefühl, das sich in Augenblicken der Einsamkeit gewissermaßen nur sich selber eingesteht, und sich in äußern Symbolen verkörpert, die das Gefühl so treu als möglich genau in der Form darstellen, in welcher es in des Dichters Geist existiert. (Hervorhebungen im Original, Mill 1874, 203)

Man fühlt sich bei Mill an Heinz Schlaffers eingangs zitierte Bestimmung von Lyrik erinnert. Mill nimmt an, dass der Dichter sich seines eigenen Publikums nicht bewusst ist, Schlaffer geht davon aus, dass das Publikum im modernen Gedicht ausgeschlossen ist. Ebenso impliziert das `Ablauschen` eine mediale Festlegung der Lyrik als lautliches Gebilde, das auch in gedruckter Form als gesprochen und abgelauscht betrachtet wird. Gleichzeitig zeigt der Vergleich mit Schlaffer, dass das Verhältnis zwischen Dichter und Publikum gegenüber Mills Entwurf verkompliziert und problematisiert worden ist: Zum einen betrachtet Schlaffer das lyrische »Ich im Gedicht« als »Gemeingut seiner Leser» (Schlaffer 2012, 117), er vermeidet also eine konsequente referenzielle Festlegung des Ichs. Damit wird die Gattung wesentlich durch die Loslösung eines Texts von Entstehungs- und Gebrauchskontexten begründet. Zudem ist der Dichter nicht wie bei Mills ein introvertierter Sprecher, vielmehr ist das Kommunikationsverhältnis gemäß Schlaffer im modernen Gedicht gestört, indem das Publikum von einer hermetisch gewordenen Dichtung ausgeschlossen wird.

Dennoch, der in der französischen Literaturwissenschaft bisweilen angetroffene Verdacht, die deutschsprachige Theorie, wenn sie von >Lyrikı spreche, beschäftige sich hauptsächlich mit dem Lyrismus (vgl. Combe 1996, 39), scheint hier neue Nahrung zu erhalten. Dieser Verdacht eines deutschen Lyrismus sollte aber mit Blick auf die theoretische Forschung jüngerer Zeit insgesamt wenigstens relativiert, wenn nicht zurückgewiesen werden. Es liegen verschiedene Vor-

3 Vgl. Rodriguez 2003, 18-20, sowie die ungewöhnlich positive Verteidigung des Lyrismus bei Maulpoix 2006.

4 Vgl. die Wortschöpfung in einem Lehrbrief von Alfred de Vigny, vgl. Vigny 1848 [1829], 22. 
schläge vor, die mit offenen Merkmalskatalogen auf die Problematik eingehen, dass Lyrik auch innerhalb der Gattungstrias verortet wird und in dieser Hinsicht potenziell auf eine Vielfalt von Formen zu beziehen ist, die nicht alle lyristisch sind. Wenn man von Definitionen absieht, die Lyrik als Residualkategorie von Verstexten betrachtet, die "nicht episch oder dramatisch sind " (Fricke und Stocker 2000, 498), dann kommen hier insbesondere Prototypendefinition in den Blick (z. B. Wolf 2005; Petzold 2012; Zettelmann 2013; Hempfer 2014).

Wolf zum Beispiel beschreibt mit neun >Tendenzen munikative Kompetenz von Autoren und Lesern, Lyrik zu erkennen (vgl. Wolf 2005, 38 f.). Eventuell kommen zu diesen Tendenzen als notwendige Merkmale >Texthaftigkeit` und `Fiktionalität ‘ hinzu, jedenfalls nennt Wolf diese als angeblich unproblematische Merkmale im Eingang seines Artikels (Wolf 2005, 23-24; vgl. hierzu auch Einleitung in diesem Band). In der Liste selbst werden aber Texthaftigkeit und Fiktionalität nicht mehr aufgegriffen, vielleicht werden sie bereits als selbstverständlich vorausgesetzt. Die Tendenzen bezeichnen demgegenüber keine notwendigen Merkmale. Zu den Tendenzen gehört zum Beispiel die Kürze, die auf eine Mehrheit lyrischer Texte zutrifft, aber bekanntermaßen weder ein hinreichendes noch ein notwendiges Merkmal von Lyrik ist und insofern - wie gesagt - lediglich eine von insgesamt neun Tendenzen bezeichnet, die gemeinsam in wechselnder Kombination den Prototypen erfassen. Allerdings sind diese neun Tendenzen von unterschiedlicher Art, wie Hempfer überzeugend darlegt (vgl. Hempfer 2014, 24-25). So hat zum Beispiel das Kriterium der Versifikation (als notwendiges und nicht hinreichendes Merkmal) einen ganz anderen Status als etwa die »existence of one seemingly unmediated consiousness or agency" (Wolf 2005, 39), da das zweite Kriterium von manchen Lyrikbewegungen wie etwa den französischen Parnassiens vermieden wurde.

Die Ansätze, Lyrik über eine Reihe von Tendenzen zu definieren, mögen intuitiv geeignet sein, die Vielfalt lyrischer Texte zu erfassen. Als Notbehelfe für Definitionsschwierigkeiten haben sie aber die Suche nach einer gültigen Definition noch nicht beendet. Man kann hier weitere Formen von Lyrikdefinitionen in Betracht ziehen (vgl. Zymner 2016), etwa pakt- oder institutionstheoretische Ansätze, und nicht zuletzt Zymners Definition von Lyrik (vgl. Zymner 2009, 140). Zymner entwickelt einen Gattungsbegriff, der insbesondere diejenigen Beispiele einbezieht, die von Prototypendefinitionen eher randständig behandelt werden. Seine Definition von Lyrik als "graphische oder phonische Repräsentation von Sprache als Sprachwerk oder Sprachkunstwerk, welche als generisches Display sprachlicher Medialität fungiert und ästhetische Evidenz prozedural konstituiert« (Zymner 2009, 140), erfasst die Gattung auf einem hohen Abstraktionsniveau. Zymners Definition setzt ästhetische Mindestansprüche, grenzt das Gelegenheitsgedicht aus, liefert aber eine Konzeption von Lyrik, die keine spezifische Adres- 
santenhaltung implizieren. Demgegenüber herrschen insgesamt adressantenorientierte Definitionsansätze immer noch vor und diese sollen im Folgenden im Zentrum stehen. ${ }^{5}$

\section{Redekriterium und Lyrik}

Unter allen Vorschlägen, Lyrik innerhalb der Gattungstrias zu profilieren, indem das Redekriterium aufgrund einer spezifischen Form des Adressanten präzisiert wird, ist Hempfers $(2014,30)$ Ansatz, einen transhistorischen Prototypen anhand einer lyrischen »Sprechsituation« $\mathrm{zu}$ entwickeln, vermutlich der theoretisch avancierteste und provokativste Vorschlag. Diese (1) »Sprechsituation« ist durch eine »Ich-Jetzt-Hier-Deixis« charakterisiert, die (2) simultan zum Sprechakt eine »besprochene Situation« konstituiert (vgl. Hempfer 2014, 34, 68 f.). Lyrik lässt sich auf diese Weise innerhalb der Gattungstrias insbesondere vom Erzählen abgrenzen: Während der Erzähler sich in seiner Rede auf ein (gegebenenfalls fiktiv) vergangenes Geschehen bezieht, entwickelt der Adressant in der Lyrik die Textwelt simultan zu seinem Sprechen. Weiter präzisiert Hempfer, dass (3) die Koinzidenz von Sprechsituation und besprochener Situation lediglich eine inszenierte sei. In diesem Sinne ist die Rede des Adressanten grundlegend zu unterscheiden von der »der Kommunikationssituation zwischen Produzent und Rezipient « (Hempfer 2014, 69). Hempfer nennt dies »Performativitätsfiktion«, die sich im lyrischen Text durch (4) eine asymmetrische Beziehung zwischen Adressant und Adressat bemerkbar macht, in der ein Adressat sich allenfalls durch das Zitat des Adres-

\footnotetext{
5 Neuere Ansätze gehen häufig vom Begriff `Sprecher` aus (vgl. hierzu Einleitung in diesem Band zur medialen Festlegung, die `Sprecher` impliziert). Auch der Begriff `lyrisches Ich` wird noch rege benutzt, obwohl seine Verwendung vielfach kritisiert wurde (vgl. Killy 1972, 4; Burdorf 2015, 192). $\mathrm{Zu}$ den kritisierten Punkten gehört, dass mit \lyrischem Ich` bisweilen eine beliebige Form von Adressanten gemeint werden kann, in anderen Fällen aber nur Adressanten in spezifisch adressantenmarkierten Subgenres. Zudem sind zum lyrischen Ich gegensätzliche Theorien zum Verhältnis zwischen Adressant und Autor entworfen worden. Es liegen durchaus Vorschläge vor, wie das lyrische Ich als begriffliches Instrument präzisiert oder gerettet werden könnte (vgl. schon Spinner 1975, aber auch Martínez 2002; Schiedermair 2004). Aber auch ein jüngerer Überblick über theoretische Konzeptionen des Lyrischen Ichs von Borkowski und Winko (2011) kommt zum Schluss, dass das lyrische Ich nur noch als »Begriff auf der Objektebene« zu behandeln, das heißt als Gegenstand der Literaturgeschichte in der Zeit zwischen 1900 und 1960 (vgl. Borkowski und Winko 2011, 61).
} 
santen (das heißt des »seigentlichen`Sprecher[s]«, Hempfer 2014, 69) äußern kann. $^{6}$

Im Sinne der Performativitätsfiktion ist also davon auszugehen, dass lediglich suggeriert wird, der Adressant sei zugleich Teil der Textwelt, ohne dies wirklich zu sein. Hempfer geht zugleich von einer logischen Unmöglichkeit aus, dass der artikulierende Adressant mit dem Autor übereinstimmen könnte (vgl. Hempfer 2014, 31). Damit wird eine gattungskonstitutive Unterscheidung eingeführt: Sobald der Autor als Adressant spricht, handelt es sich nicht mehr um Lyrik. Dieser Fall wäre zum Beispiel bei einem Gelegenheitsgedicht gegeben, bei dem ein Autor als »ich« sich auf eine reale Situation bzw. Gelegenheit bezieht. Die Performativitätsfiktion setzt demnach für Lyrik eine unüberbrückbare ontologische Grenze zwischen Textwelt und Diskurswelt voraus, und hieraus folgt eine grundlegende Fiktionalität der Gattung.

An Hempfers Betonung einer grundlegenden Unterscheidung von Adressant und Autor lässt sich also die These der grundsätzlichen Dekontextualisierung oder Fiktionalisierung von Lyrik festmachen. Das heißt, dass ein Text in einem bestimmten Kontext als Gebrauchstext lediglich ein Gedicht ist, aber zur Lyrik werden kann, sofern er losgelöst von diesem Kontext lesbar wird. Dass unter dieser Prämisse ein Text unter bestimmten Umständen Lyrik und unter anderen Bedingungen Nicht-Lyrik sein soll, erscheint mir kontra-intuitiv. Vor allem ist dies ein Beleg für Virginia Jacksons These, dass `Lyrikı eine Gattung geworden sei, die nahelegt, Verstexte notwendigerweise von ihren Entstehungs- und Gebrauchskontexten abgelöst zu lesen. Da Hempfer in dieser Hinsicht insbesondere die realweltliche Referenz blockiert sieht, ist in diesem Fall tatsächlich von einer Fiktionalisierung der Gattung auszugehen. Dies wäre eine schwerwiegende Interpretationshypothese, deren Anwendung auf einen Verstext im Prinzip immer wieder kritisch geprüft werden müsste.

Eine solche Fiktionalität ist aber in der Theorie längst nicht selbstverständlich. So hat Lamping fiktionale bzw. nicht-fiktionale Rede und fiktiven bzw. nicht-fiktiven Inhalt differenziert (vgl. Lamping 1989, 102-110). Man könnte hier ebenso Zymners Unterscheidung von sautor-fiktional/faktual und spersonafiktional/faktualı anführen (vgl. Zymner 2009, 10-11).

Eine noch weitreichendere Position hat Culler (2015) formuliert, die nur oberflächlich dem Hempfer'schen Ansatz ähnlich ist. Culler argumentiert ebenso

6 Der seigentliche Sprecher« muss wohl im Sinne von Hempfer als der »dominante Sprecher« des Gedichts betrachtet werden, das heißt es wird hier keine Stufung der Kommunikation vorgeschlagen, sondern in der prototypischen Lyrik sind kleinere Gegenreden als abhängig vom dominanten Sprecher zu betrachten. 
prototypenorientiert wie Hempfer, er ist insofern vorsichtiger, als er bei der Lyrik von einer durch die Produzenten und Rezipienten etablierten Tradition ausgeht, die gewissermaßen die Gattung historisch rückwärts projiziert. ${ }^{7}$ Der »enunciative apparatus« sieht bei Culler $(2015,34)$ neben Adressanten explizit Adressaten sowie ein Publikum als optionale Aspekte einer lyrischen Äußerungsstruktur vor (vgl. Culler 2015, 206).

Der auffälligste Unterschied zwischen Culler und Hempfer betrifft aber die Position des Autors im Verhältnis zum Adressanten, denn den kommunikativen Akt des Adressanten versteht Culler (2015, 127-128) im Anschluss an Hamburger (1977) als »Wirklichkeitsaussage«. Culler begründet diese Position im Rahmen des rhetorischen Konzepts der Epideixis, der Demonstration kunstfertiger Rede. Der Adressant (»speaker«) mache Wertaussagen über die (Diskurs-)Welt (»claims [...] about our world«, Culler 2015, 128) und biete einen auf sperlokutionäre Effekte beim Rezipienten ausgelegten Text. Unter >perlokutionären Effekten` sind grundlegende Wirkungsweisen auf den Rezipienten zu verstehen im Sinne von Rühren oder gar Überzeugen.

Der Bezug auf Rhetorik, Epideixis und Wertpostulate dient bei Culler dazu, den Fiktionalitätsaspekt der Lyrik zu relativieren. In den Beispielen nimmt aber Culler wenig Bezug auf die Frage, auf welche Weise solche Wirklichkeitsaussagen sich konstituieren. Bei der Behandlung von Goethes »Heidenröslein«, das mit Präteritum und einem basalen Plot durchaus balladesk-erzählende Elemente aufweist (»Sah ein Knab’ [...]/ Lief er schnell [...]«), wird sichtbar, dass Wirklichkeitsaussagen auch das Resultat einer Allegorese sein können. Epideixis lässt sich dann so verstehen, dass Goethe als Autor verantwortlich ist für eine Wirklichkeitsaussage, die im vorliegenden Fall in fiktionaler Form formuliert ist. Das ist im Grunde genommen die in der deutschsprachigen Forschung bereits vielfach kritisierte Position von Hamburger, der zufolge das Lyrische Ich immer ein reales Aussagesubjekt sei (vgl. Hamburger 1977, 217). Bei Culler ist diese Position etwas relativiert durch die Tatsache, dass er die Möglichkeit eines fiktionalen Sprechers zumindest akzeptiert, auch wenn dieser Fall ihn augenscheinlich nicht sonderlich interessiert. Dennoch ist das »Heidenröslein«-Beispiel so zu verstehen, dass der Inhalt des Gedichts zwar fiktiv ist, der Adressant aber mit Goethe gleichgesetzt werden könne. Tatsächlich weisen in diesem Gedicht keine Deiktika auf einen Adressanten hin, explizit äußern sich nur die fiktiven Figuren.

7 Allerdings führt er das Fehlen einer Lyriktheorie auf eine Unterlassung von Aristoteles zurück (vgl. Culler 2015, 72) und liefert damit Hempfer die zentralen Voraussetzungen für den transhistorischen Prototypen (vgl. Hempfer 2014, 10). Zeitlich ist das insofern möglich, als Culler die wesentlichen Grundsätze seines Buchs zuvor in Artikeln veröffentlicht hatte. 
Sowohl Culler (2015) als auch Hempfer (2014) legen prototypisch strukturierte Gattungskonzepte vor und diskutieren jeweils eine mögliche Öffnung des Prototyps in Richtung dramatischem oder narrativem Modus. Culler und Hempfer unterscheiden sich aber im Punkt der Fiktionalität des Adressanten. Und sie kommen $\mathrm{zu}$ diesen unterschiedlichen Schlüssen anhand eines ziemlich ähnlichen Korpus von hochkanonischen Beispielen der Lyrik. Dabei ist auffällig, wie sehr die Ansätze bei ähnlichen Interessen und einem, wie man meinen möchte, gemeinsamen Gegenstand divergieren. Dies ist besorgniserregend, wenn man vom didaktischen Anspruch ausgeht, dass solche Theorien auch Modelle für eine angemessene und wertschätzende Lektüre von Lyrik zu Verfügung stellen sollen. Immerhin lassen solche Divergenzen annehmen, dass zumindest manche Gattungsdefinition unter Umständen unangemessene Interpretationsverfahren nahelegen.

Wie angemessen sind solche Annahmen von grundlegenden Äußerungsstrukturen der Lyrik, wie sie bei Hempfer oder Culler aufgezeigt werden? Bezieht man den Fragekomplex etwa auf ein Beispiel von gnomischer Lyrik, dann produziert meines Erachtens der Ansatz von Hempfer insofern unplausible Ergebnisse, als die Texte entweder unnötig fiktionalisiert werden oder aus der Gattung ausscheiden. Man betrachte zum Beispiel das Einleitungsgedicht von Annette von Droste-Hülshoffs Erzählung Die Judenbuche:

\author{
Wo ist die Hand so zart, daß ohne Irren \\ Sie sondern mag beschränkten Hirnes Wirren, \\ So fest, daß ohne Zittern sie den Stein \\ Mag schleudern auf ein arm verkümmert Seyn? \\ Wer wagt es, eitlen Blutes Drang zu messen, \\ $\mathrm{Zu}$ wägen jedes Wort, das unvergessen \\ In junge Brust die zähen Wurzeln trieb, \\ Des Vorurtheils geheimen Seelendieb? \\ $\mathrm{Du}$ Glücklicher, geboren und gehegt \\ Im lichten Raum, von frommer Hand gepflegt, \\ Leg hin die Waagschal', nimmer dir erlaubt! \\ Lass ruhn den Stein - er trifft dein eignes Haupt! (Droste-Hülshoff 1842, 381)
}

Das Gedicht steht in der Ausgabe des Morgenblatts für gebildete Leser direkt unter dem Titel vor der Erzählung, es teilt sich den Titel gewissermaßen mit der nachfolgenden Erzählung. Das Gedicht ist mit fünfhebigen Jamben, sechs Paarreimen, die im ersten und dritten Verspaar mit klingenden Kadenzen enden, einfach gebaut. Der Inhalt lässt sich leicht zurückführen auf die Rede von Jesus (Joh. 8,7) bei der Konfrontation mit der Ehebrecherin, die den Rahmen für den Spruch bietet, dass, wer ohne Sünde sei, den ersten Stein werfe. Das Interessante sind die Abweichungen gegenüber dem Evangelium. Im Evangelium spricht Jesus 
über eine Ehebrecherin, im vorliegenden Fall macht die Autorin eine Aussage, die sich auf den männlichen Übeltäter Friedrich Mergel bezieht. Sind die männlichen und weiblichen Rollen hier vertauscht? Nicht ganz, mit >zartı, >fest ‘ und ১fromm ‘ bezieht sich zum Beispiel die Hand (V. 1, 3 und 10) metonymisch auf weiblich und männlich konnotierte Rollen. Hingegen ist der Adressat (»Du Glücklicher«, V. 9) männlich.

Die Textwelt ist minimal ausgestattet. Die ersten vier Verspaare stellen lediglich Suchfragen. Erst gegen Ende taucht ein Du auf, das immerhin auch ein Ich impliziert. Es scheint also kein adressantenfreies Gedicht zu sein, auch wenn der Adressant linguistisch ansonsten wenig Spuren hinterlässt. Wollte man den Text mit Hempfer als Lyrik lesen (wobei es ungewiss ist, ob Hempfer dies tatsächlich unterstützen würde), wäre anzunehmen, dass ein Adressant hier Fragen und einen abschließenden, biblisch inspirierten Weisheitsspruch an einen Adressaten richtet. In einem weiteren Schritt könnte man dann sogar noch die Frage aufwerfen, inwiefern der Adressant mit dem nicht-diegetischen Erzähler identisch sein könnte. Plausibler scheint mir allerdings die Annahme, dass der Adressant einen ergänzenden erbaulichen Kommentar zur Erzählung an den Leser im äußeren Kommunikationsrahmen richtet. »Du« ist in diesem Sinne der jeweilige Leser, der Adressant wiederum drückt in allgemeinen Worten die Werthaltung der Autorin aus. ${ }^{8}$ Tatsächlich könnte man meines Erachtens mit gutem Recht die Frage stellen, inwiefern eine Unterscheidung von Adressant und Autorin einen Gewinn für das Verständnis des Texts verspricht. Im Sinne von Hempfer wäre dann aber der Schluss zu ziehen, dass es sich nicht mehr um Lyrik handelt. Dies erscheint mir als ein problematisches Ergebnis, da Gattungszuordnung und interpretative Konsequenzen in einem hohen Maße in ein gegenseitiges Abhängigkeitsverhältnis treten.

\section{Sprechhaltungen als Tendenzen der Lyrik}

Angesichts der gegensätzlichen Bestimmungen von Culler (2015) und Hempfer (2014) erscheint es sinnvoll, die Definition von Lyrik nicht von einem eingeschränkten Typus einer Adressantenhaltung abhängig zu machen. Tatsächlich gibt es

8 Hier wäre, aus der Sicht von Schlaffer und verschiedenen Exponenten der Theorie des >lyrischen Ichs die Frage zu stellen, inwiefern die Aussage vom Rezipienten übernommen werden kann (vgl. ebenso Rabaté 2013, 13). Hier ist auffällig, dass Hempfer zum `Sprecher eine passive Hörerkonzeption postuliert. Die Dekontextualisierung impliziert also bei Hempfer keine völlige Lockerung der Referenz des `Sprechers`. 
auch verschiedene Vorschläge, wie Lyrik mit unterschiedlichen Äußerungsstrukturen in Verbindung gebracht werden könnte. Petzold (2012) bietet eine in vielerlei Hinsicht erweiterte Fassung von Wolfs (2005) Prototypenmodell an, ergänzt dieses um ein narratologisch inspiriertes, gestuftes Kommunikationsmodell und behandelt auf dieser Grundlage die Frage der Äußerungsstruktur differenzierter. Die Tendenzen der Lyrik umfassen bei Petzold insbesondere eine »typische Kommunikationsstruktur«. Diese kann auf vielfältige Weise realisiert werden, müsse aber in jedem Fall »als Ergebnis eines fiktionalen Sprechakts verstanden« werden (vgl. Petzold 2012, 115 und 148). Die Fiktionalität scheint allerdings in diesem Fall fast gleichbedeutend mit Vermitteltheit zu sein, sie ist also zugleich Ausdruck der Auffassung, dass in lyrischen Texten immer ein »Sprecher « gegeben ist. Im Weiteren stellt Petzold vor allem die Vielfältigkeit der Kommunikationsstrukturen heraus. Modellhaft spricht er von einer »Ebene der primären Sprechinstanz«, die sich »häufig als >Ich` im Text« manifestiert (Petzold 2012, 142). Mit inbegriffen ist die Möglichkeit einer ıscheinbaren Sprecherlosigkeit` (vgl. Petzold 2012, 181). Denkbar ist zudem, dass in Abhängigkeit von der primären Sprechinstanz eine sekundäre Sprechinstanz vorliegt (vgl. Petzold 2012, 151), die man aber meines Erachtens auch als Figurenrede verstehen könnte. Insgesamt gilt aber der primäre Sprecher als Produzent des Gedichttexts. Paratexte wie Titel können aber nicht unbedingt der primären Sprechinstanz zugerechnet werden. Ebenso könnten die expliziten Aussagen des primären Sprechers von einer impliziten Bedeutung ironisch konterkariert werden (vgl. Petzold 2012, 154-155). Petzold verzichtet an dieser Stelle auf den aus der Narratologie geläufigen Begriff des simplizierten Autors « und geht von einer konstruktivistischen Projektion direkt auf den Autor aus (vgl. Petzold 2012, 156). Es kann also zusammengefasst werden, dass Petzold in jedem Fall von der Präsenz eines Adressanten ausgeht, auch wenn dieser im Text nicht an linguistischen Markern festgemacht werden kann. Hypothetische Rückschlüsse auf die Autorintention sind gleichwohl möglich. Problematisch an der Konzeption scheinen mir auch in diesem Fall die notwendige Unterstellung von Fiktionalität und der Zwang, in jedem Gedicht einen vom Autor abstrahierten Adressanten zu finden (vgl. Einleitung zu diesem Band).

Der Autor-Bezug lässt sich vielleicht im Rückgriff auf Borkowski und Winko (2011) ausführen. Mit dem Ziel, den Begriff des ılyrischen Ichs` durch eine Taxonomie von unterschiedlichen Sprecherpositionen zu ersetzen, unterscheiden Borkowski und Winko einerseits einen referenziellen Aspekt, andererseits einen kontextuellen Aspekt der Frage >Wer spricht das Gedicht?^. In referenzieller Hinsicht liegen im Grunde genommen drei Optionen vor: Der textinterne »Sprecher 1 « als »Urheber der Äußerungen, die den Gedichttext ausmachen « (Borkowski und Winko 2011, 64), „Sprecher 2 « als reale Kommunikationsinstanz und es gilt schließlich den Fall zu bedenken, dass alle Fragen nach der Referenz nicht 
mit dem Bezug auf eine Sprechinstanz beantwortet werden können (vgl. Borkowski und Winko 2011, 76). Man sieht, dass bei Borkowskis und Winko ssprechen nicht immer dasselbe bedeutet. Bei der realen Kommunikationsinstanz ist ein tatsächliches Laut-Geben gemeint, beim textinternen Sprecher 1 ist das >Sprechen eine Illusion des schriftlichen Texts. Wie nun die Referenz auf allfällige Sprecher im Gedicht vollzogen wird oder nicht, hängt gemäß Borkowski und Winko von kontextuellen Faktoren ab (das heißt von der sprachlichen Faktur des Textes, Bedingungen der Rezeption oder auch institutionellen Vorgaben). Diese kontextuellen Faktoren können jeweils nahelegen, einen Sprecher 1 oder 2 auf den Autor zu beziehen oder nicht, wenn aber alle diese Referenzannahmen ohne Erfolg bleiben, dann liegt keine Sprechinstanz vor und das Gedicht bezieht sich direkt auf den Autor als Urheber, nicht als Sprecher (vgl. Borkowski und Winko 2011, 76-77).

Zunächst ist festzuhalten, dass Borkowski und Winko bewusst vermeiden, die Gattung Lyrik aufgrund von spezifischen Adressantenhaltungen oder Äußerungsstrukturen zu definieren. Dies ist angesichts derzeit widersprechender Definitionen von Lyrik zu begrüßen. Weiter ist hervorzuheben, dass mit dem »Sprecher 2 « die wichtige Frage der Performanz aufgegriffen wird. In dieser Hinsicht ist der Begriff des eigentlichen `Sprechers ‘ für die Lyrik von zunehmender Bedeutung, da mit dem Internet auditive oder audio-visuelle Aufnahmen von Lyrik eine zunehmende Verbreitung erhalten haben.

\section{Schluss}

Die vorangehende Darstellung hat gezeigt, dass zur Frage von Fiktionalität und Formen der Adressanten in der Lyrik immer noch konträre Auffassungen bestehen. Fiktionalität wird der Lyrik aus unterschiedlichen Gründen zugesprochen. Bei Petzold (2012) gründet die Annahme in der angeblich notwendigen Präsenz einer Vermittlungsinstanz. Hempfer begründet die Performativitätsfiktion mit der letztlich unmöglichen Koinzidenz des Verfassens eines Gedichttexts und seiner simultanen Artikulation. In allen diskutierten Fällen scheint es aber insbesondere darum zu gehen, dass Lyrik sich grundsätzlich durch eine Dekontextualisierung und Ablösung von realweltlicher Referenz auszeichnet. Während man solche Effekte in vielen lyrischen Texten erkennen kann, wird mit dieser Bestimmung zugleich eine ganze Reihe von Gedichtformen wie Epigramm oder Gelegenheitsgedicht von der Gattung ausgeschlossen. Problematisch hieran wäre aber insbesondere, dass das eine oder andere Gelegenheitsgedicht oder Gedankengedicht wieder aufgenommen werden kann, wenn es sich denn im Hempfer'schen Sinne 
lyrisch dekontextualisiert lesen lässt. Dies wäre eine Entscheidung für einen spezifisch ästhetischen Lyrikbegriff, dessen transhistorischer Anspruch meines Erachtens nicht überzeugend erfüllt werden kann.

Auf dem gegenwärtigen Stand der Debatte erscheint es aber sowieso verfrüht, vermutlich auch insgesamt ungünstig, die Definition der Lyrik mit einer spezifischen Äußerungsstruktur zu verknüpfen. Immerhin gibt es ernstzunehmende Vorschläge, wie derjenige von Borkowski und Winko (2011), lyrische Texte zuzulassen, deren Adressanten auf den Autor bzw. die als Text adressantenfrei direkt auf den Autor verweisen. Aufgrund einer spezifischen Adressanten-Konstellation sollte man jedenfalls derzeit auf eine Aussage über die Zugehörigkeit oder NichtZugehörigkeit zur Gattung verzichten. Eine Festschreibung der Gattung auf eine notwendig fiktionale Adressanteninstanz wäre nicht zuletzt eine bedauerliche Einschränkung der Lyrikologie. ${ }^{9}$ Dessen ungeachtet könnte die Forschung zu einem Konsens kommen, `Gedicht` und `Lyrikı auch in der Gegenwartsliteratur wieder stärker zu differenzieren (wie dies zum Beispiel Lamping 1989 vorgeschlagen hat). In diesem Fall wäre eine Einschränkung der Gattung Lyrik auf eine spezifische Äußerungsstruktur zumindest begründbar, aber vermutlich müsste man dann davon absehen, den Begriff im Rahmen der Gattungstrias zu verwenden. Vermutlich würde dies >Lyrikı auf ein Korpus einschränken, das bezüglich Versform und Inhalt ziemlich gut den Erwartungen an die Gattung Lyrik entspricht, mit denen auch Studierende an die Universitäten kommen, aber wenig mit den Entwicklungen der Gegenwartsdichtung zu tun hat.

Eine andere Frage ist, ob man überhaupt von der Vorstellung einer Kommunikation in der Lyrik Abschied nehmen sollte (etwa nach dem Muster eines Kommunikationsmodells mit Sender, Botschaft und Empfänger, vgl. Petzold 2012, 138-140). Aus lyrikologischer Sicht hat zum Beispiel Zymner vorgebracht, dass literarische Texte süblicherweise noch eine geteilte, geschlossene Kommunikationssituation etablieren (vgl. Zymner in diesem Band). Ein eigentliches Verbot, im Zusammenhang von Lyrik nicht an eine Form von Kommunikation zu denken, erscheint mir angesichts der Tatsache, dass es durchaus Theorieentwürfe von Kommunikation gibt, die ohne eine Mechanik von Sender-Botschaft-Empfänger auskommen (z. B. Sperber und Wilson 1995), übertrieben. Kommunikation kann zum Beispiel mit Sperber und Wilson (1995) letztlich auch als eine Form der Aufmerksamkeitslenkung anderer

9 Mit einem Seitenblick auf die gegenwärtige Auseinandersetzung um `erzählerloses Erzählen` oder transmediales Erzählen in der Narratologie (vgl. z. B. Nünning und Nünning 2002; Patron 2009; Kindt und Köppe 2014) scheint es auch nicht gerade wünschenswert, den Gegenstand der Lyrikologie von einer bestimmten Form der Vermitteltheit abhängig zu machen. 
verstanden werden. Der Adressant wäre in diesem Sinne eine Instanz, die mithilfe von Sprache nicht unbedingt eine Botschaft übermittelt, aber vielleicht auf etwas aufmerksam macht - und sei es, dass das Gebilde auf die impliziten Regeln von Lyrik aufmerksam macht, indem es diese untererfüllt, wie Handkes »Die Aufstellung des 1. FC Nürnberg vom 27.1.1968«.

\section{Literaturverzeichnis}

Borkowski, Jan und Simone Winko. „Wer spricht das Gedicht? noch einmal zum Begriff lyrisches Ich und zu seinen Ersetzungsvorschlägen." Lyrische Narrationen - narrative Lyrik.

Gattungsinterferenzen in der mittelalterlichen Literatur. Hg. Hartmut Bleumer und Caroline Emmelius. Berlin: de Gruyter, 2011. 43-77.

Burdorf, Dieter. Einführung in die Gedichtanalyse. 3. Aufl. Stuttgart: Metzler, 2015.

Combe, Dominique. "La référence dédoublée. Le sujet lyrique entre fiction et autobiographie." Figures du lyrique, Hg. Dominique Rabaté. Paris: Presses Universitaires de France, 1996. 39-63.

Culler, Jonathan. Theory of the Lyric. Cambridge, MA.: Harvard Univ. Press, 2015.

Droste-Hülshoff, Annette von. „Die Judenbuche. Ein Sittengemälde aus dem gebirgigten Westphalen." Morgenblatt für gebildete Leser 36.96 (22. April 1842): 381-382.

Fricke, Harald und Peter Stocker. »Lyrik. « Reallexikon der deutschen Literaturwissenschaft, Hg. Harald Fricke in Zusammenarbeit mit Georg Braungart, Klaus Grubmüller, Jan-Dirk Müller, Friedrich Vollhardt und Klaus Weimar , 2. Aufl. Berlin: de Gruyter, 2000. 498-502.

Hamburger, Käte. Die Logik der Dichtung. 3. Aufl. Stuttgart: Klett-Cotta, 1977.

Hempfer, Klaus W. Lyrik. Skizze einer systematischen Theorie. Stuttgart: Franz Steiner, 2014.

Huss, Bernhard, Florian Mehltretter und Gerhard Regn. Lyriktheorie(n) der italienischen Renaissance. Berlin: de Gruyter, 2012.

Jackson, Virginia. Dickinson's Misery. A Theory of Lyric Reading. Princeton: Princeton Univ. Press, 2005.

Jackson, Virginia und Yopie Prins. "General Introduction. "The Lyric Theory Reader. A Critical Introduction, Hg. Virginia Jackson und Yopie Prins. Baltimore: Johns Hopkins Univ. Press, 2014. 1-8.

Killy, Walter. Elemente der Lyrik. München: Beck, 1972.

Kindt, Tom und Tilmann Köppe. Erzähltheorie. Eine Einführung. Stuttgart: Reclam, 2014.

Lamping, Dieter. Das lyrische Gedicht. Definitionen zu Theorie und Geschichte der Gattung. Göttingen: Vandenhoeck und Ruprecht, 1989.

Martínez, Matías. »Das lyrische Ich. Verteidigung eines Begriffs. «Autorschaft. Positionen und Revisionen, Hg. Heinrich Detering. Stuttgart: Metzler, 2002. 376-389.

Maulpoix, Jean-Michel. »énonciation et élévation. Deux remarques sur le lyrisme. « Lyrisme et énonciation lyrique, Hg. Nathalie Watteyne. Québec: Éditions Nota Bene, Presses Univ. de Bordeaux, 2006. 29-37.

Mill, John Stuart. »Gedanken über Poesie und ihre verschiedenen Arten [1833]. « August Comte und der Positivismus. Vermischte Schriften. Hg. Elise Gomperz. Leipzig: Fues's Verlag, 1874. 197-222. 
Nünning, Vera und Ansgar Nünning. Erzähltheorie transgenerisch, intermedial, interdisziplinär. Trier: Wissenschaftlicher Verlag Trier, 2002.

Opitz, Martin. Buch von der Deutschen Poeterey [1624]. Stuttgart: Reclam, 1977.

Patron, Sylvie. Le narrateur. Introduction à la théorie narrative. Paris: Colin, 2009.

Petzold, Jochen. Sprechsituationen lyrischer Dichtung. Ein Beitrag zur Gattungstypologie. Würzburg: Königshausen \& Neumann, 2012.

Primavesi, Oliver. „Aere perennius? Die Antike Transformation der Lyrik und die neuzeitliche Gattungstrinität. "Sprachen der Lyrik. Von der Antike bis zur digitalen Poesie. Für Gerhard Regn anlässlich seines 60. Geburtstags, Hg. Klaus W. Hempfer. Stuttgart: Steiner, 2008. $15-32$.

Rabaté, Dominique. Gestes lyriques. Paris: Corti, 2013.

Rodriguez, Antonio. Le pacte lyrique. Configuration discursive et interaction affective. Sprimont: Mardaga, 2003.

Schiedermair, Simone. \Lyrisches Ich und sprachliches sichı. Literarische Funktionen der Deixis. München: iudicium, 2004.

Schlaffer, Heinz. Geistersprache. Zweck und Mittel der Lyrik. München: Hanser, 2012.

Sperber, Dan und Deidre Wilson. Relevance. Communication and Cognition. 2. Aufl. Malden: Blackwell, 1995.

Spinner, Kaspar H. Zur Struktur des lyrischen Ich. Frankfurt a. M.: Akad. Verlagsgesellschaft, 1975.

Vigny, Alfred. »Lettre à Lord ${ }^{\star \star \star}$, sur la soirée du 24 octobre 1829, et sur un système dramatique." Théâtre complet du Comte Alfred de Vigny. Paris: Charpentier, 1848. 10-27.

Wellek, René. »Genre Theory, the Lyric, and Erlebnis [1967]." The Lyric Theory Reader. A Critical Introduction, Hg. Virginia Jackson und Yopie Prins. Baltimore: Johns Hopkins Univ. Press, 2014. 40-52.

Wolf, Werner. «The Lyric. Problems of Definition and a Proposal for Reconceptualisation.» Theory into Poetry. New Approaches to the Lyric, Hg. Eva Müller-Zettelmann und Margarete Rubik. Amsterdam: Rodopi, 2005. 21-56.

Zettelmann, Eva. »Lyrik und kulturelles Gedächtnis. « Geschichtslyrik. Ein Kompendium, Hg. Heinrich Detering und Peer Trilcke. Göttingen: Wallstein, 2013. 141-172.

Zymner, Rüdiger. Lyrik. Umriss und Begriff. Paderborn: Mentis, 2009.

Zymner, Rüdiger. »Theorien der Lyrik seit dem 18. Jahrhundert.« Handbuch Lyrik. Theorie, Analyse, Geschichte Hg. Dieter Lamping. 2. Aufl. Stuttgart: Metzler, 2016. 23-36. 
